\section{European Medical Research Group (Meeting held on 6 November 1990)}

The European Medical Research Group met at the Medical Society of London, Lettsom House, on 6 November 1990. The guest speaker was Professor Stafford Lightman, of the Department of Medicine, Charing Cross Hospital, London, who gave a lecture on 'New insight into hypothalamic function'.

Following a period of questions and discussion, a poster session was held demonstrating the research in progress of some members of the Group. Their abstracts are published below.

\section{Selective increase in type II muscle turnover as an explanation of wasting in inflammatory bowel disease}

\section{A.K. Banerjee, V.R. Preedy and T.J. Peters}

Departments of Surgery and Clinical Biochemistry, King's College Hospital, London SE5 9PJ, UK.

Muscle wasting and negative nitrogen balance are common features of severe inflammatory bowel disease (IBD) in man. This is commonly attributed to malnutrition and malabsorption, but the mechanism is poorly understood. We have established a rat model of IBD and determined protein synthetic rate in the various fibre types in this model. The experimental group of 5 week old male Sprague-Dawley rats $(n=9)$ were fed indomethacin $8 \mathrm{mg} / \mathrm{kg} /$ day for 3 days mixed with chow, whilst controls were fed chow alone. Following the feeding period both groups were fed normal diet for 4 days, when the rats were killed and protein synthetic rate in the plantaris (type II), soleus (type I) and gastrocnemius (mixed) muscle fractions were determined by a labelled phenylalanine flooding dose technique, as previously described. ${ }^{1}$

The plantaris demonstrated a significant $10 \%$ increase in protein synthesis [control 15.3(2.0), experimental 16.7(0.192) g protein/day/g DNA:mean (s.d.); $P<0.05$, ANOVAR]. Thus, experimental non-steroidal antiinflammatory drug (NSAID) enteropathy is causing, as an early effect, selective type II fibre dysfunction, as manifested by both increased stromalprotein synthesis and increased whole protein turnover. The exact mechanism is unclear, but the effect may be profoundly relevant in the study of the early consequences of both NSAID enteropathy and IBD, in general, on protein turnover in man. Similar mechanisms have been postulated in the pathogenesis of muscular dystrophy. ${ }^{2}$ Furthermore, the studies may also help to explain the clinical observation of persistent muscle and skeletal weakness in arthritic patients maintained on NSAID treatment, even when overt joint inflammation has been controlled.

\section{References}

1. Preedy, V.R. \& Sugden, P.H. The effects of fasting or hypoxia on rates of protein synthesis in vivo in subcellular fractions of rat heart and gastrocnemius muscle. Biochem J 1989, 257: 519-527.

2. Maclennan, P.A. \& Edwards, R.H.T. Abs. Clin Sci 1990, 78 (Suppl 22): 1P.

Differential gut protein synthesis in a non steroidal anti-inflammatory model of inflammatory bowel disease

A.K. Banerjee, J.S. Marway, V.R. Preedy and T.J. Peters

Departments of Surgery and Clinical Biochemistry, King's College Hospital Medical School, London, UK.

Abnormalities in gut mucosal protein turnover represent a potential mechanism for both the pathogenesis and the development of complications, such as stricturing, in inflammatory bowel disease. We have established an experimental indomethacin-induced rat model of ileitis characterized by early increases in permeability and biochemical abnormalities progressing into ulcers, skip lesions, chronic inflammation and strictures. Using this model we examined protein synthesis and turnover. The experimental group of 5 week old male Sprague-Dawley rats $(n=9)$ were fed indomethacin $8 \mathrm{mg} / \mathrm{kg} / \mathrm{day}$ for 3 days mixed with a powdered form of chow. The control group of rats were fed the same diet for 3 days without indomethacin. Following the feeding period both groups were fed normal solid diet for 4 days recording daily body weight and food intake. At the end of this period the development of enteropathy was tested by the in vivo ${ }^{51} \mathrm{Cr}$ EDTA test, the animal killed, and protein synthesis, DNA and RNA turnover determined by a labelled phenylalanine flooding dose technique as described elsewhere. ${ }^{1,2}$

Animals with enteropathy have a significantly increased rate of protein synthesis in the proximal ileum, compared with controls [control 40.9(12.4), enteropathic 48.4(9.6) $\mathrm{mg}$ protein/day:mean (s.d.); $P<0.03, t$-test] but not the duodenum, although indomethacin is known to cause superficial ulcers in the latter site. These changes may reflect altered neutrophil protease activity, and may also partly explain the development of strictures in both NSAID enteropathy and IBD in general.

\section{References}

1. Banerjee, A.K. \& Peters, T.J. The pathogenesis of Crohn's disease. Lancet 1989, ii: 1459-1460.

2. Preedy, V.R., Paska, L., Sugden, P.H., Schofield, P.S. \& Sugden, M.C. Protein synthesis in liver and extra hepatic tissues after partial hepatectomy. Biochem J 1990, 267: 325-330. 
Oesophagitis associated with chronic non-steroidal anti-inflammatory drug use - an under-diagnosed problem?

\section{G.L. Swift, J.D. Arnold and J. Rhodes}

University Hospital of Wales, Cardiff, Wales, UK.

Damage of the gastric and duodenal mucosa from both short-term and chronic administration of non-steroidal anti-inflammatory drugs (NSAID) is well recognized. Endoscopic findings correlate poorly with symptoms and the risk of gastrointestinal haemorrhage cannot be predicted. There is little information on the effect of NSAID therapy on the oesophagus although an increased incidence of benign oesophageal strictures is reported. Fifty patients ( 28 female) with rheumatoid arthritis were randomly selected from rheumatology clinics. All had been taking indomethacin for at least 1 year. Endoscopy was performed and oesophageal findings graded according to modified Savary and Miller criteria. Lesions in the stomach and duodenum were also noted. Endoscopy was normal in 12 subjects. Oesophageal lesions were present in $10(20 \%)$ : these were erythema in 3 , linear erosions in 5 and ulcers in 2. Gastric erosions were present in 13 and gastric ulcers in 3 and 12 patients had duodenal erythema \pm erosions.

This high incidence of asymptomatic oesophageal disease should be considered in long-term management of patients on NSAIDs. 